\title{
Enhancing Cell-Free Massive MIMO networks through LEO Satellite Integration
}

\author{
F. Riera-Palou*, G. Femenias*, M. Caus ${ }^{\dagger}$, M. Shaat ${ }^{\dagger}$, J. García-Morales* $^{*}$ A. I. Pérez-Neira ${ }^{\dagger}$ \\ *Mobile Communications Group - Universitat de les Illes Balears - 07122 Mallorca, Spain \\ ${ }^{\dagger}$ Centre Tecnològic de Telecomunicacions de Catalunya (CTTC/CERCA) - 08860 Barcelona, Spain \\ Email: \{felip.riera,guillem.femenias,jan.garcia\}@uib.es, \{marius.caus,musbah.shaat,ana.perez\}@cttc.es
}

\begin{abstract}
This paper proposes a hybrid network architecture combining a cell-free Massive MIMO terrestrial layout with a low Earth orbit satellite segment jointly targeting the maximization of the minimum per-user rate in the coverage area. Towards this end, an optimization framework is proposed, alongside a greedy solution, that diverts the terrestrial users experiencing poor propagation conditions to the satellite segment. Simulation results show the substantial benefits this integrated approach brings along, very specially when the terrestrial segment operates single-antenna access points or is sparsely deployed.

Index Terms-Cell-free, Massive MIMO, Low Earth orbit (LEO), Hybrid networks, Max-min performance.
\end{abstract}

\section{INTRODUCTION}

Despite not being a priority in the initial $5 \mathrm{G}$ studies, the incorporation of a satellite segment into the $5 \mathrm{G}$ ecosystem has recently gained momentum [1], [2], specially with the advent of low Earth orbit (LEO) satellites. Owing to their much lower altitude in comparison to classical geostationary orbits, communications using LEO satellites are subject to low latencies with round-trip delays in the range of 30-100 ms (rivalling those found in terrestrial networks) and the possibility of combined terrestrial-satellite terminals able to directly communicate with both the ground and the satellite segments [3], [4] are pushing the move to integrated space-terrestrial architectures. As an example, authors in [5], [6] have recently introduced data-offloading schemes from the terrestrial to a LEO-based satellite segment with the objective of maximizing the spectral and energy efficiencies of the whole network. In contrast, the ultradense network paradigm was one of the pivotal concepts in the genesis of 5G and one that will surely keep playing a fundamental role in the future evolution of $5 \mathrm{G}$ towards beyond $5 \mathrm{G}$ (B5G)/6G systems. One specific flavour of network densification that has attracted considerable attention is the cell-free massive MIMO (CF-M-MIMO) architecture. Initially proposed in [7], [8], CF-M-MIMO assumes the existence of a

Work supported by MINECO under project TERESA-TEC201790093-C3-1/3-R (AEI/FEDER,UE), Spain. single central processing unit (CPU) to which a plethora of access points (APs), irregularly distributed over the area to be covered, are connected via fronthaul links. In practice, there are limits on the geographical area a single CF-M-MIMO network can cover and the most likely scenario is that of several CF-M-MIMO networks, each covering a relatively large area (a few to tens of square kilometers), among which some degree of coordination is exerted. Interestingly, by applying a socalled max-min power control strategy, CF-M-MIMO is able to provide a uniform quality-of-service (QoS) throughout the coverage area resulting in all mobile stations (MSs) attaining the same rate. However, a well-known caveat of max-min network optimization is that the performance of the whole network may be seriously compromised by a few ill-conditioned users that bring down the common user rate, a fact that has been shown to occur in both centralized massive multiple-input multiple-output (M-MIMO) [9] as well as decentralized CF-M-MIMO [10]. It was already pointed out in [11] that the advent of LEO constellations is likely to make this space-based solution significantly more cost effective and flexible than the classical fiber-linked macrocellular architecture. Towards this end, this paper aims at combining these two promising B5G/6G trends, namely, satellite integration and cell-free topology. In particular, a two-tier architecture is proposed where a large number of CF-M-MIMO terrestrial segments, each targeting a densely populated zone, is backed by a constellation of LEO satellites that can serve a double purpose: firstly, to provide coverage continuity across large terrestrial hotspots and, secondly, to serve as an alternative link for ill-conditioned terrestrial users [10].

\section{SYSTEM MODEL}

We consider an scenario such as the one depicted in Fig. 1 whereby several geographically separated cell-free networks are being coordinated by a base station controller (BSC) that, in turn, is collocated with the gateway to a multibeam LEO-based satellite segment (SAT-GW). 


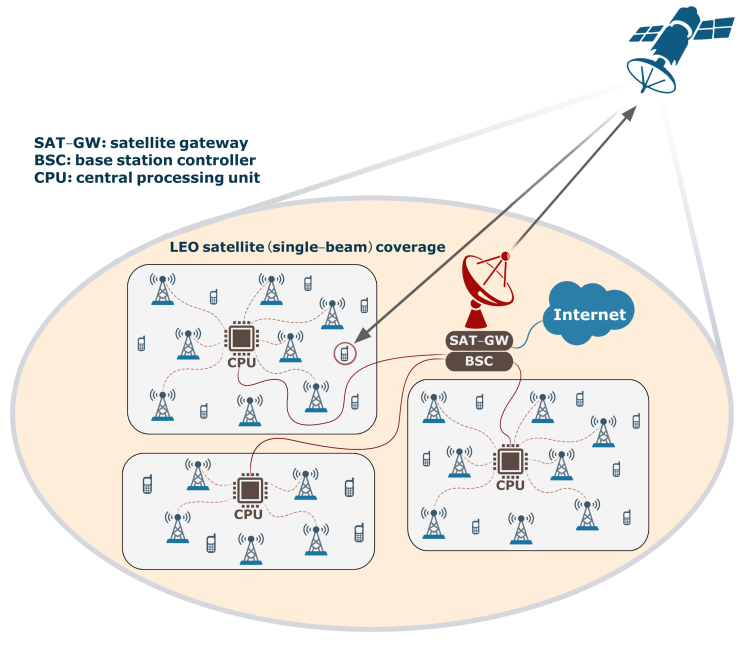

Fig. 1: Hybrid cell-free/LEO-based satellite network.

Both network elements, BSC and SAT-GW, have Internet access and it is assumed that they are coordinated so that both segments can be jointly managed at these points (e.g., MSs can be moved from one segment to the other) as postulated in the EU H2020-SANSA project and in other terrestrial-satellite integrated proposals [12]. In line with most previous literature, each CF-M-MIMO network provides service to areas in the order of a few square $\mathrm{km}$, whereas the LEO satellite coverage is in the order of several thousands of square $\mathrm{km}$, thus ensuring that the satellite illuminates the area potentially occupied by a few hundred CF-M-MIMO deployments, thus it is safe to assume that a single beam will be able to cover several CF-M-MIMO networks. As it can be observed in Fig. 1, each CF-M-MIMO deployment consists of many APs, each with available transmit power $P_{T}^{\mathrm{AP}}$, randomly distributed (locally) following a uniform distribution and connected through fronthaul links (dashed lines) to a CPU, which in turn is connected to the BSC/SATGW node through backhaul links (solid lines). In line with the original spirit of CF-M-MIMO systems, each CF-M-MIMO segment targets the provision of equal quality-of-service (QoS) to the MSs it is serving locally. For simplicity of exhibition, throughout the rest of the paper, we will concentrate on the performance of one arbitrary CF-M-MIMO network consisting of $M$ APs each equipped with $N^{\mathrm{AP}}$ antennas that jointly provide service to $K$ single-antenna MSs randomly distributed throughout the coverage area of that particular CF-MMIMO (rounded boxes in Fig. 1). It is nevertheless worth pointing out that the coordination of disjoint CFM-MIMO deployments has been recently investigated in [10], [13]. The role the satellite segment can play in such coordination constitutes a promising area for further research. The LEO satellite is located at height $h^{\mathrm{SAT}}$ above the Earth, with typical values in the range $300 \leq h^{\mathrm{SAT}} \leq 2000 \mathrm{~km}$ and it has available per-beam transmit power $P_{T}^{\mathrm{SAT}}$. It is assumed that the satellite antenna architecture has a single feed per beam, and therefore to all modelling effects can be considered a single-antenna system.

\section{A. Channel models and estimation}

Denote by $\beta_{m k}^{\mathrm{CF}}$ the large-scale propagation losses (i.e., path loss and shadowing) of the link joining AP $m$ and MS $k$, which can be expressed as $\beta_{m k}^{\mathrm{CF}}=\zeta_{m k} \chi_{m k}^{\mathrm{CF}}$ with $\zeta_{m k}$ representing the distance-dependent path loss

$$
\zeta_{m k}[\mathrm{~dB}]=\zeta_{0}+10 \alpha \log _{10}\left(d_{m k}\right),
$$

where $\zeta_{0}$ is the path loss at a reference distance of 1 $\mathrm{m}, d_{m k}$ is the distance from AP $m$ to MS $k$ and $\alpha$ is the path loss exponent. These two coefficients may vary under line-of-sight (LOS) and non-line-of-sight (NLOS) conditions. Variable $\chi_{m k}^{\mathrm{CF}}$ corresponds to the shadow fading component modeled as a correlated log-normal random variable $(\mathrm{RV})$ with zero mean and variance $\sigma_{\chi}^{2}$ whose spatial correlation model is described in [7, (54)(55)]. The link between the $m$-th AP and the $k$-th MS will be considered to be either in LOS or NLOS, with the LOS probability following

$$
p_{\mathrm{LOS}}\left(d_{m k}\right)=\min \left(1, \frac{d_{0}}{d_{m k}}+\left(1-\frac{d_{0}}{d_{m k}}\right) e^{-\frac{d_{m k}}{2 d_{0}}}\right),
$$

where $d_{0}$ is a reference distance governing the LOS/NLOS transition.

The resulting downlink channel vector $\boldsymbol{g}_{m k} \in \mathbb{C}^{N^{\mathrm{AP}} \times 1}$ from the $k$-th MS to the $m$-th AP (including both largescale and small-scale fading) can then be generically characterized as a Ricean fading channel consisting of a LOS component on top of a Rayleigh distributed component [14]. That is,

$$
\boldsymbol{g}_{m k}=\sqrt{\frac{K_{m k}}{K_{m k}+1}} \overline{\boldsymbol{h}}_{m k}+\sqrt{\frac{1}{K_{m k}+1}} \boldsymbol{h}_{m k},
$$

with $\overline{\boldsymbol{h}}_{m k}=\sqrt{\beta_{m k}^{\mathrm{CF}}} \boldsymbol{a}_{m k}$, where vector

$$
\boldsymbol{a}_{m k}=\left[1, e^{j \pi \sin \psi_{m k}}, \cdots, e^{j\left(N_{a p}-1\right) \pi \sin \psi_{m k}}\right]^{T}
$$

is the array response at the $\mathrm{AP}$ with $\psi_{m k}$ denoting the angle of arrival between the $k$-th MS and the $m$-th AP. The NLOS component $\boldsymbol{h}_{m k}$ follows a distribution $\mathcal{C N}\left(\mathbf{0}, \boldsymbol{R}_{m k}\right)$ with $\boldsymbol{R}_{m k}$ representing the spatial correlation of the antenna array at AP $m$ as seen from user $k$, modelled as in [15, Chapter 2], and subject to $\operatorname{Tr}\left(\boldsymbol{R}_{m k}\right)=\beta_{m k}^{\mathrm{CF}} / N^{\mathrm{AP}}$. Parameter $K_{m k}$ denotes the 
Ricean $K$-factor, with $K_{m k}=0$ for NLOS propagation links and $10 \log _{10}\left(K_{m k}\right) \sim \mathcal{N}\left(\mu_{K}, \sigma_{K}^{2}\right)$ for LOS propagation links. It can be shown that a channel conforming to (2) is distributed as $\boldsymbol{g}_{m k} \sim \mathcal{C N}\left(\tilde{\overline{\boldsymbol{h}}}_{m k}, \tilde{\boldsymbol{R}}_{m k}\right)$, where

$\tilde{\overline{\boldsymbol{h}}}_{m k}=\sqrt{\frac{K_{m k}}{K_{m k}+1}} \overline{\boldsymbol{h}}_{m k}$ and $\tilde{\boldsymbol{R}}_{m k}=\boldsymbol{R}_{m k} /\left(K_{m k}+1\right)$.

The equivalent satellite-to-MS channel is modelled using a scalar gain $v_{k}$ characterized by a Ricean distribution conforming to the specifications in [16],

$$
v_{k}=\sqrt{\frac{K_{k}}{K_{k}+1}} \bar{v}_{k}+\sqrt{\frac{\beta_{k}^{\mathrm{SAT}}}{K_{k}+1}} \breve{v}_{k},
$$

where $\breve{v}_{k}$ is the multipath (flat fading) component with distribution $\mathcal{C N}(0,1), K_{k}$ is the $k$-th satellite-user Ricean $K$-factor, and $\bar{v}_{k}$ is the direct path given by

$$
\bar{v}_{k}=\sqrt{\beta_{k}^{\mathrm{SAT}}} e^{j \theta_{k}^{\mathrm{LOS}}} \quad \text { with } \quad \beta_{k}^{\mathrm{SAT}}=\frac{G_{R} G_{T}^{k}}{L_{k}} \chi_{k}^{\mathrm{SAT}},
$$

where $\theta_{k}^{\mathrm{LOS}}$ represents the phase term that results from the beam radiation pattern and the radiowave propagation in the direct path, and $\beta_{k}^{\text {SAT }}$ models the large-scale propagation losses (pathloss and shadowing) with $G_{R}$ denoting the MS antenna gain and $G_{T}^{k}$ corresponds to the satellite antenna gain in the direction of the $k$-th user position. The shadowing component $\chi_{k}^{\mathrm{SAT}}$ is a log-normal random variable with mean and variance $\left(\mu_{k}, \vartheta_{k}^{2}\right)$ whose specific values depend on whether the specific user experiences good or bad propagation conditions. The Ricean $K$-factors for each user, $K_{k}$, are assumed to conform to a log-normal distribution whose mean and variance are specified in $[16,6.7 .1]$ again in accordance to the user's good/bad status. Finally, $L_{k}$ represents the large-scale propagation losses (due to free-space propagation) that are defined as $L_{k}=10^{L_{k}^{\mathrm{dB}} / 10}$ with $L_{k}^{\mathrm{dB}}$ denoting the losses of the Friis' model in $\mathrm{dB}$ [16]

$$
L_{k}^{\mathrm{dB}}=32.45+20 \log _{10}\left(f_{c}^{\mathrm{SAT}}\right)+20 \log _{10}\left(d_{k}^{\mathrm{SAT}}\right),
$$

with $f_{c}^{\mathrm{SAT}}$ denoting the carrier frequency (in $\mathrm{GHz}$ ) of the satellite component and $d_{k}^{\mathrm{SAT}}$ being the distance (in $\mathrm{m})$ separating the satellite from user $k$. Note that the MS-to-satellite distance can now be computed as [16]

$d_{k}^{\mathrm{SAT}}=\sqrt{R_{E}^{2} \sin \varpi^{2}+\left(h^{\mathrm{SAT}}\right)^{2}+2 h^{\mathrm{SAT}} R_{E}}-R_{E} \sin \varpi$, where $R_{E}$ is the Earth's radius and $\varpi$ denotes the elevation angle from the CF-M-MIMO network to the LEO satellite. Regarding the fast fading term, this is generated in accordance to the two-state model specified in [16] whereby users are considered to be in either good or bad states, assumed here to correspond to the probability of being in LOS/NLOS scenario as defined in Table 6.6.1-1 in [16]. It is now easy to check that the satellite channel follows a distribution

$$
v_{k} \sim \mathcal{C N}\left(\sqrt{\frac{K_{k}}{K_{k}+1}} \bar{v}_{k}, \frac{\beta_{k}^{\mathrm{SAT}}}{K_{k}+1}\right) .
$$

Two conditions are assumed: 1) as in most previous literature, the LMS channel is safely assumed to be frequency flat, and 2) the channel is deemed constant during a frame transmission. In addition, receivers are provisioned with satellite ephemeris and are equipped with GNSS receivers, thus the MSs are capable of compensating Doppler effects.

During the uplink (UL) training phase, all $K$ MSs simultaneously transmit pilot sequences of $\tau_{p}$ samples to the APs and thus, the $N \times \tau_{p}$ received UL signal matrix at the $m$ th active AP is given by

$$
\boldsymbol{Y}_{p_{m}}=\sqrt{\tau_{p} P_{p}^{\mathrm{AP}}} \sum_{k=1}^{K} \boldsymbol{g}_{m k} \boldsymbol{\varphi}_{k}^{T}+\boldsymbol{N}_{p_{m}}
$$

where $P_{p}^{\mathrm{AP}}$ is the available pilot symbol power, $\boldsymbol{\varphi}_{k}$ denotes the $\tau_{p} \times 1$ training sequence assigned to $\mathrm{MS}$ $k$, with $\left\|\boldsymbol{\varphi}_{k}\right\|^{2}=1$, and $\boldsymbol{N}_{p_{m}} \in \mathbb{C}^{N \times \tau_{p}}$ is a matrix of independent identically distributed (iid) zero-mean circularly symmetric Gaussian random variables with standard deviation $\sigma_{u}$. Standard results on CF-M-MIMO (see [14] for details) show that the MMSE channel estimate, $\hat{\boldsymbol{g}}_{m k}$, is distributed as

$$
\hat{\boldsymbol{g}}_{m k} \sim \mathcal{C N}\left(\tilde{\overline{\boldsymbol{h}}}_{m k}, \boldsymbol{\Gamma}_{m k}\right)
$$

where $\boldsymbol{\Gamma}_{m k}=\tau_{p} P_{p}^{\mathrm{AP}} \tilde{\boldsymbol{R}}_{m k} \boldsymbol{\Psi}_{m k}^{-1} \tilde{\boldsymbol{R}}_{m k}^{H}$ and with matrix $\boldsymbol{\Psi}_{m k}=\tau_{p} P_{p}^{\mathrm{AP}} \sum_{k^{\prime}=1}^{K} \tilde{\boldsymbol{R}}_{m k^{\prime}}\left|\boldsymbol{\varphi}_{k^{\prime}}^{H} \boldsymbol{\varphi}_{k}\right|^{2}+\sigma_{u}^{2} \boldsymbol{I}_{N}$. The channel estimation error, $\boldsymbol{\varepsilon}_{m k}=\boldsymbol{g}_{m k}-\hat{\boldsymbol{g}}_{m k}$, conforms to a distribution $\boldsymbol{\varepsilon}_{m k} \sim \mathcal{C N}\left(\mathbf{0}, \boldsymbol{A}_{m k}\right)$ with $\boldsymbol{A}_{m k}=$ $\tilde{\boldsymbol{R}}_{m k}-\boldsymbol{\Gamma}_{m k}$. The $M N_{\mathrm{AP}} \times 1$ vector collecting the channel responses from all APs to user $k$ in the network is defined as $\boldsymbol{g}_{k}=\left[\boldsymbol{g}_{1 k}^{T} \ldots \boldsymbol{g}_{M k}^{T}\right]^{T}$ and its corresponding estimate as $\hat{\boldsymbol{g}}_{k}$. The $M N^{\mathrm{AP}} \times K$ matrix $\mathbf{G}=\left[\boldsymbol{g}_{1} \ldots \boldsymbol{g}_{K}\right]$ collects the channels between the $M$ APs and the $K$ MSs and $\hat{\mathbf{G}}$ denotes its MMSE estimate. Owing to its frequency-flat character, channel estimation of the satellite-MS link is conducted by transmitting a unit norm pilot symbol $\phi_{k}$ with power $P_{p}^{\mathrm{SAT}}$ from the satellite and performing matched filtering at the MS,

$$
y_{k}^{\mathrm{SAT}}=\sqrt{P_{p}^{\mathrm{SAT}}} v_{k} \phi_{k} \phi_{k}^{*}+e_{k}^{\mathrm{sat}} \phi_{k}^{*},
$$

with reception noise $e_{k}^{\text {sat }} \sim \mathcal{C N}\left(0, \sigma_{e}^{2}\right)$. Assuming as in the CF-M-MIMO segment that the LOS component is perfectly known, the MMSE channel estimate follows

$$
\hat{v}_{k}=\sqrt{\frac{K_{k}}{K_{k}+1}} \bar{v}_{k}+\frac{\beta_{k}^{\mathrm{SAT}} \sqrt{P_{p}^{\mathrm{SAT}}}}{\xi_{k}\left(K_{k}+1\right)} \tilde{y}_{k}^{\mathrm{SAT}}
$$


with $\xi_{k}=\frac{P_{p}^{\mathrm{SAT}} \beta_{k}^{\mathrm{SAT}}}{K_{k}+1}+\sigma_{e}^{2}$ and $\tilde{y}_{k}^{\mathrm{SAT}}=y_{k}^{\mathrm{SAT}}-\sqrt{\frac{P_{p}^{\mathrm{SAT}} K_{k}}{K_{k}+1}} \bar{v}_{k}$. This estimate can easily be shown to be distributed as $\hat{v}_{k} \sim \mathcal{C N}\left(\sqrt{\frac{K_{k}}{K_{k}+1}} \bar{v}_{k}, \frac{P_{p}^{\mathrm{SAT}}\left(\beta_{k}^{\mathrm{SAT}}\right)^{2}}{\xi_{k}\left(K_{k}+1\right)^{2}}\right)$. For later convenience, we define the channel estimation error as $\epsilon_{k}=\hat{v}_{k}-v_{k}$ which has mean $E\left\{\epsilon_{k}\right\}=0$ and variance

$$
\sigma_{\epsilon, k}^{2}=\frac{\beta_{k}^{\mathrm{SAT}}}{K_{k}+1}-\frac{P_{p}^{\mathrm{SAT}}\left(\beta_{k}^{\mathrm{SAT}}\right)^{2}}{\xi_{k}\left(K_{k}+1\right)^{2}} .
$$

\section{B. Precoding schemes}

Let us assume that the set of $K$ users to be serviced, denoted by $\mathcal{U}$, can be split into two subsets $\mathcal{U}^{\mathrm{AP}}$ and $\mathcal{U}^{\mathrm{SAT}}$ with cardinalities $K^{\mathrm{AP}}$ and $K^{\mathrm{SAT}}$, which denote the MSs served by the terrestrial APs and the LEO satellite, respectively. Precoding at the CF-M-MIMO segment relies on zero-forcing $(\mathrm{ZF})$, which is conducted at the CPU. In particular, at each signaling interval, the CPU jointly processes the $K^{\mathrm{AP}} \times 1$ vector $\boldsymbol{q}_{\mathrm{AP}}=$ $\left[q_{1}^{\mathrm{AP}} \ldots q_{K^{\mathrm{AP}}}^{\mathrm{AP}}\right]^{T}$ of information symbols to be transmitted through the terrestrial segment using linear precoding as

$$
\boldsymbol{x}^{\mathrm{AP}}=\left[\left(\boldsymbol{x}_{1}^{\mathrm{AP}}\right)^{T} \ldots\left(\boldsymbol{x}_{M}^{\mathrm{AP}}\right)^{T}\right]^{T}=\mathbf{W}_{\mathrm{AP}} \mathbf{P}_{\mathrm{AP}}^{1 / 2} \boldsymbol{q}_{\mathrm{AP}},
$$

where the $M N^{\mathrm{AP}} \times K^{\mathrm{AP}}$ matrix $\mathbf{W}_{\mathrm{AP}}$ corresponds to the $\mathrm{ZF}$ precoding operation that is given by

$$
\mathbf{W}_{\mathrm{AP}}=\hat{\mathbf{G}}_{\mathrm{AP}}^{H}\left(\hat{\mathbf{G}}_{\mathrm{AP}} \hat{\mathbf{G}}_{\mathrm{AP}}^{H}\right)^{-1},
$$

with $\hat{\mathbf{G}}_{\mathrm{AP}}$ collecting the columns of $\hat{\mathbf{G}}$ corresponding to the MSs in $\mathcal{U}^{\mathrm{AP}}$, and $\mathbf{P}_{\mathrm{AP}}=\operatorname{diag}\left(\left[\eta_{1}^{\mathrm{AP}} \ldots \eta_{K^{\mathrm{AP}}}^{\mathrm{AP}}\right]\right)$ is a power allocation diagonal matrix ${ }^{1}$ with $\eta_{k}^{\mathrm{AP}}$ denoting the power coefficient applied to an arbitrary user $k^{\mathrm{AP}} \in \mathcal{U}^{\mathrm{AP}}$. From the implementation point of view, the $M N^{\mathrm{AP}} \times 1$ vector $\boldsymbol{x}_{\mathrm{AP}}$ is computed at the CPU with each $N^{\mathrm{AP}} \times 1$ sub-vector, $\boldsymbol{x}_{m}^{\mathrm{AP}}$, then being forwarded to the $m$ th AP.

The estimated symbol by $\mathrm{MS} k^{\mathrm{AP}} \in \mathcal{U}^{\mathrm{AP}}$ is

$$
\hat{q}_{k^{\mathrm{AP}}}=\mathbf{g}_{k^{\mathrm{AP}}}^{T} \boldsymbol{x}_{\mathrm{AP}}+w_{k^{\mathrm{AP}}},
$$

with $w_{k^{\mathrm{AP}}} \sim \mathcal{C N}\left(0, \sigma_{w, \mathrm{AP}}^{2}\right)$ denoting the receiver additive white Gaussian noise (AWGN) sample. A tight lower bound on the achievable rate for user $k^{\mathrm{AP}}$ is

$$
R_{k^{\mathrm{AP}}}=B_{\mathrm{AP}} \frac{1-\tau_{p} / \tau_{c}}{2} \log _{2}\left(1+\mathrm{SINR}_{k^{\mathrm{AP}}}\right),
$$

where $\mathrm{SINR}_{k^{\mathrm{AP}}}$ constitutes an approximation to the average SINR of the $k$ th-user and is given $\mathrm{by}^{2}$ [8]

$$
\operatorname{SINR}_{k^{\mathrm{AP}}}=\frac{P_{T}^{\mathrm{AP}} \eta_{k^{\mathrm{AP}}}^{\mathrm{AP}}}{P_{T}^{\mathrm{AP}} \sum_{k^{\prime}=1}^{K^{\mathrm{AP}}} \gamma_{k^{\mathrm{AP}} k^{\prime}} \eta_{k^{\prime}}^{\mathrm{AP}}+\sigma_{w, \mathrm{AP}}^{2}},
$$

${ }^{1} \mathcal{D}(\mathbf{A})$ denotes the vector formed by the main diagonal of matrix $\mathbf{A}$ and $\operatorname{diag}(\boldsymbol{x})$ is a diagonal matrix with vector $\boldsymbol{x}$ at its main diagonal.

${ }^{2}$ Different CF-M-MIMO segments are sufficiently apart so as to neglect their interference. where $\gamma_{k k^{\prime}}$ is the $k^{\prime}$ th entry in vector

$$
\gamma_{k}=\mathcal{D}\left(E\left\{\left(\hat{\mathbf{G}}^{T} \hat{\mathbf{G}}^{*}\right)^{-1} \hat{\mathbf{G}}^{T} \boldsymbol{A}_{m k} \hat{\mathbf{G}}^{*}\left(\hat{\mathbf{G}}^{T} \hat{\mathbf{G}}^{*}\right)^{-1}\right\}\right)
$$

Note in (5) that $B_{\mathrm{AP}}$ denotes the system bandwidth used by the CF-M-MIMO component and the term $1-\tau_{p} / \tau_{c}$ represents the rate losses due to the training phase with the factor $1 / 2$ accounting for the equal time/frequency division between uplink and downlink.

Following [8], the minimum achievable rate among all users in the CF-M-MIMO segment can be maximized by solving the following optimization problem

$$
\begin{aligned}
& \max \min _{\eta_{1}^{\mathrm{AP}}, \ldots, \eta_{K^{\mathrm{AP}}}^{\mathrm{AP}}} \operatorname{SINR}_{k^{\mathrm{AP}}} \quad \forall k^{\mathrm{AP}} \in \mathcal{U}^{\mathrm{AP}} \\
& \text { s.t. }\left|\boldsymbol{x}_{m}^{\mathrm{AP}}\right|^{2} \leq P_{T}^{\mathrm{AP}} \quad \forall m \in\{1, \ldots, M\},
\end{aligned}
$$

which can be shown to be a quasi-convex optimization problem solvable using an iterative algorithm based on a bisection search [8]. Due to the monotonic relation between rate and SINR, maximizing the minimum SINR also implies de maximization of the minimum rate $R_{k^{\mathrm{AP}}} \forall k^{\mathrm{AP}} \in \mathcal{U}^{\mathrm{AP}}$. A key point to note now is that the common SINR that results from optimizing (7) is greatly conditioned by the worst users in $\mathcal{U}^{\mathrm{AP}}$, which can significantly lower the overall network performance.

It is assumed that satellite diverted users share the available bandwidth $B_{\mathrm{SAT}}^{\mathrm{DL}}$ in an (O)FDMA fashion. Considering that an arbitrary satellite-serviced user is indexed by $k^{\mathrm{SAT}}$, the transmitted symbol from the satellite conforms to $x_{k^{\mathrm{SAT}}}=\sqrt{\eta_{k^{\mathrm{SAT}}}^{\mathrm{SAT}}} q_{k^{\mathrm{SAT}}}$ with $q_{k^{\mathrm{SAT}}}$ representing the corresponding information symbol and $\eta_{k}^{\mathrm{SAT}}$ the power weight. At the reception end, the user terminal implements a matched filter to maximize the received SINR and, relying on the fact that $\hat{v}_{k^{\mathrm{SAT}}}=v_{k^{\mathrm{SAT}}}+\epsilon_{k^{\mathrm{SAT}}}$, allows the user estimated symbol to be expressed as

$\hat{q}_{k^{\mathrm{SAT}}}=\sqrt{\eta_{k^{\mathrm{SAT}}}^{\mathrm{SAT}}} q_{k^{\mathrm{SAT}}}^{\mathrm{SAT}}\left(\left|\hat{v}_{k^{\mathrm{SAT}}}\right|^{2}+\hat{v}_{k^{\mathrm{SAT}}}^{*} \epsilon_{k^{\mathrm{SAT}}}\right)+\hat{v}_{k^{\mathrm{SAT}}}^{*} w_{k^{\mathrm{SAT}}}$,

with $w_{k^{\text {SAT }}}$ denoting a zero-mean AWGN sample with variance $\sigma_{w, \mathrm{SAT}}^{2}$. Based on the previous equation, the instantaneous SINR for user $k^{\mathrm{SAT}} \in \mathcal{U}^{\mathrm{SAT}}$ follows

$$
\operatorname{SINR}_{k^{\mathrm{SAT}}}^{\text {inst }}=\frac{\eta_{k^{\mathrm{SAT}}}^{\mathrm{SAT}}\left|\hat{v}_{k^{\mathrm{SAT}}}\right|^{2}}{\eta_{k^{\mathrm{SAT}}}^{\mathrm{SAT}}\left|\epsilon_{k^{\mathrm{SAT}}}\right|^{2}+\sigma_{w, \mathrm{SAT}}^{2}},
$$

from which, relying on Jensen's inequality, an achievable rate for this satellite user can then be derived as

$$
R_{k^{\mathrm{SAT}}}=\frac{B_{\mathrm{SAT}}^{\mathrm{DL}}}{K^{\mathrm{SAT}}} \log _{2}\left(1+\mathrm{SINR}_{k^{\mathrm{SAT}}}\right)
$$

where $B_{\mathrm{SAT}}^{\mathrm{DL}}$ is the total bandwidth available for downlink satellite transmission noting that this has to be shared 
among all the $K^{\mathrm{SAT}}$ users diverted to the satellite segment and $\operatorname{SINR}_{k} \mathrm{SAT}=E\left\{\operatorname{SINR}_{k}^{\text {inst }}\right\}$ is bounded by

$$
\operatorname{SINR}_{k} k^{\mathrm{SAT}}=\frac{\eta_{k^{\mathrm{SAT}}}^{\mathrm{SAT}} E\left\{\left|\hat{v}_{k^{\mathrm{SAT}}}\right|^{2}\right\}}{\eta_{k^{\mathrm{SAT}}}^{\mathrm{ST}} E\left\{\left|\epsilon_{k^{\mathrm{SAT}}}\right|^{2}\right\}+\sigma_{w, \mathrm{SAT}}^{2}},
$$

which builds on the Gaussian hypothesis assuming on the independence between numerator and denominator. An $\mathrm{SINR}_{k}$ SAT closed-form expression is obtained noting that $E\left\{\left|\epsilon_{k^{\mathrm{SAT}}}\right|^{2}\right\}=\sigma_{\epsilon, k}^{2}$ (see (4)) and

$$
E\left\{\left|\hat{v}_{k^{\mathrm{SAT}}}\right|^{2}\right\}=\frac{K_{k}}{K_{k}+1} \beta_{k}^{2}+2 \frac{P_{p}^{\mathrm{SAT}}\left(\beta_{k}^{\mathrm{SAT}}\right)^{2}}{\xi_{k}\left(K_{k}+1\right)^{2}} .
$$

In line with the terrestrial segment, power loads are chosen so as to maximize the minimum average SINR (i.e., rate) of the users served by the satellite. Formally,

$$
\begin{aligned}
& \max \underset{\eta_{1}^{\mathrm{SAT}}, \ldots, \eta_{K^{\mathrm{SAT}}}^{\mathrm{SAT}}}{\operatorname{SINR}_{k} \mathrm{SAT}} \quad \forall k^{\mathrm{SAT}} \in \mathcal{U}^{\mathrm{SAT}} \\
& \text { s.t. } \sum_{k \in \mathcal{U}^{\mathrm{SAT}}} \eta_{k}^{\mathrm{SAT}}=P_{T}^{\mathrm{SAT}},
\end{aligned}
$$

where the constraint takes into account the total transmission power $P_{T}^{\mathrm{SAT}}$. Problem (9) has the same form as that in (7) and can be solved using the same strategy.

\section{LEO-ENHANCED CELL-FREE OPERATION}

Despite the dispersion of a large number of APs throughout the coverage area favours this uniform quality of service (QoS) behaviour, it is often the case that a few ill-positioned users bring down the performance of the whole network, a situation often found in LOS environments [9]. Even though discarding these bad users has the potential to significantly increase the network capacity [17], it is not advisable from an operator point of view to simply leave users unserved and therefore, alternatives need to be found. Towards this end, this paper advocates for the off-loading of the users that limit the performance of the terrestrial network towards the satellite segment. In doing so, we merge problems (7) and (9) while still keeping the requirement of maximizing the minimum rate (or SINR) of any user in the coverage area.

The max-min LEO-enhanced CF-MIMO optimization problem can be formally posed as

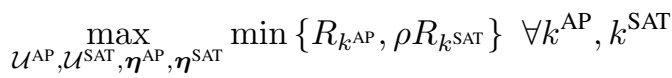

$$
\begin{aligned}
& \text { s.t. }\left|\boldsymbol{x}_{m}^{\mathrm{AP}}\right|^{2} \leq P_{T}^{\mathrm{AP}} \quad \forall m \text { and } \sum_{k \in \mathcal{U}^{\mathrm{SAT}}} \eta_{k}=P_{T}^{\mathrm{SAT}} \text {, }
\end{aligned}
$$

where $\rho$, with $0 \leq \rho \leq 1$, is a designer chosen weighing parameter that serves to bias the optimization to favour the use of the terrestrial segment (in particular, when $\rho=0$, the proposed hybrid system totally neglects the

\section{$\overline{\text { Algorithm } 1 \text { Enhanced max-min power/user allocation. }}$}

Inputs: $\rho, \gamma_{k}, \beta_{m k}^{\mathrm{CF}}, \beta_{k}^{\mathrm{SAT}} \forall m, k$.

Initialization:

1) Selected users/index: $\mathcal{U}_{(0)}^{\mathrm{AP}}=\mathcal{U}, \mathcal{U}_{(0)}^{\mathrm{SAT}}=\emptyset, i=0$.

2) Compute, solving (7), optimum max-min power coefficients $\eta_{k^{\mathrm{AP}}}^{(0)}$ for $k^{\mathrm{AP}} \in \mathcal{U}_{(0)}^{\mathrm{AP}}$.

3) Initial mininum rate $R_{\text {min }}^{(0)}=\min \left\{R_{k^{\mathrm{AP}}}^{(0)}\right\} \quad \forall k^{\mathrm{AP}}$. Repeat

1) Select worst user $\tilde{k} \in \mathcal{U}_{(i)}^{\mathrm{AP}}$ : $\tilde{k}=\arg \min _{k \in \mathcal{U}_{(i)}^{\mathrm{AP}}} f\left(\left\{\beta_{m k}^{\mathrm{CF}}\right\}\right)$ for $m \in\{1, \ldots, M\}$.

2) Update iteration: $i=i+1$.

3) Update user subsets: $\mathcal{U}_{(i)}^{\mathrm{AP}}=\mathcal{U}_{(i-1)}^{\mathrm{AP}}-\{\tilde{k}\}$, $\mathcal{U}_{(i)}^{\mathrm{SAT}}=\mathcal{U}_{(i-1)}^{\mathrm{SAT}} \cup\{\tilde{k}\}$.

4) Recompute, solving (7), optimum max-min power

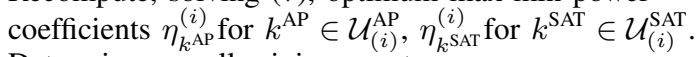

5) Determine overall minimum rate $R_{\text {min }}^{(i)}=\min \left\{R_{k^{\mathrm{AP}}}^{(i)}, \rho R_{k^{\mathrm{SAT}}}^{(i)}\right\} \quad \forall k^{\mathrm{AP}}, k^{\mathrm{SAT}}$.

until $R_{\min }^{(i)} \leq R_{\min }^{(i-1)}$ or $i=K^{\mathrm{AP}}$

Outputs: Selected user sets $\mathcal{U}_{\mathrm{opt}}^{\mathrm{AP}}=\mathcal{U}_{(i-1)}^{\mathrm{AP}}, \mathcal{U}_{\mathrm{opt}}^{\mathrm{SAT}}=\mathcal{U}_{(i-1)}^{\mathrm{SAT}}$ and power coefficients $\boldsymbol{\eta}_{(i-1)}^{\mathrm{AP}}, \boldsymbol{\eta}_{(i-1)}^{\mathrm{SAT}}$.

satellite segment and thus becomes a conventional CFM-MIMO network ((10) falls back to (7)). Problem (10) is a constrained mixed-integer optimization problem and, as such, it is non-convex and its solution requires of an exhaustive search over all possible user groupings in $\mathcal{U}^{\mathrm{AP}}$ and $\mathcal{U}^{\text {SAT }}$. Such a search quickly becomes unfeasible even for a modest number of users given that the evaluation of each possible combination entails solving the max-min optimization problems given by (7) and (9). Fixing sets $\mathcal{U}^{\mathrm{AP}}$ and $\mathcal{U}^{\mathrm{SAT}}$ convexifies the problem with respect to variables $\boldsymbol{\eta}^{\mathrm{AP}}$ and $\boldsymbol{\eta}^{\mathrm{SAT}}$ and its solution can proceed by solving (7) and (9) independently. Given the NP character of (10), we tackle it using a computationally viable greedy approach, detailed in Algorithm 1 , to be executed at the BSC/SAT-GW. In particular, we take as starting point of the search a setup where all users are served through the CF-M-MIMO network (i.e., $\mathcal{U}^{\mathrm{AP}}=\mathcal{U}, \mathcal{U}^{\mathrm{SAT}}=\emptyset$ ). At each step, a user is transferred from the terrestrial to the satellite segment and the objective function in (10) is recalculated. Ideally, the transferred user is selected as the one maximizing $\min \left\{R_{k^{\mathrm{AP}}}, \rho R_{k^{\mathrm{SAT}}}\right\}$. The procedure finalizes whenever the terrestrial-to-satellite offloading of an additional user leads to a degradation of the minimum rate across all users in both segments. User re-assignment is done on a large-scale basis by solely using large-scale parameters $\left(\beta_{m k}^{\mathrm{CF}}, \Gamma_{k}\right)$ that are always available at the BSC/SAT-GW.

It is important to recognize that step 1 of the loop, where the worst user in $\operatorname{set} \mathcal{U}_{(i)}^{\mathrm{AP}}$ is to be elicited, invari- 
ably requires evaluating (5) and (8) for each possible user and the corresponding terrestrial to satellite relocation. In order to simplify this selection and following results in [17], the user to be relocated is determined solely based on the large-scale propagation gains $\beta_{m k^{\mathrm{AP}}}$. To this end the function $f\left(\left\{\beta_{m k^{\mathrm{AP}}}\right\}\right)$ in step 1 of the loop can be any function that maps the large-scale gains from a given CF-M-MIMO user to all APs, to a scalar metric that somehow summarizes how well positioned is that user in the serviced area. Among several functions that have been evaluated, the one leading to best results is to use as channel quality metric for a user $k^{\mathrm{AP}} \in \mathcal{U}^{\mathrm{AP}}, f\left(\left\{\beta_{m k^{\mathrm{AP}}}\right\}\right)=\max _{m}\left\{\beta_{1 k^{\mathrm{AP}}}, \ldots, \beta_{M k^{\mathrm{AP}}}\right\}$. Consequently, the diverted user is the one whose maximum (terrestrial) large-scale gain is minimum.

\section{NUMERICAL RESUlTS}

We consider a downlink scenario where a single CFM-MIMO terrestrial network is providing coverage to an squared area of side $L$ through the random (uniform) deployment of $M$ multi-antenna APs, each with $P_{t}^{\mathrm{AP}}=200 \mathrm{~mW}$. The terrestrial segment is supplemented by a LEO satellite situated at a height of $600 \mathrm{~km}$ above the Earth with an elevation angle of $\varpi=30^{\circ}$, thus resulting in a satellite to CF-M-MIMO distance of $1,075 \mathrm{~km}$. The satellite transponder is assumed to have multibeam capabilities and to be equipped with directional antennas with a maximum gain of 30.5 $\mathrm{dBi}$. The satellite link operates at a carrier frequency of $20 \mathrm{GHz}$ (Ka-band) over a per-beam bandwidth of $B^{\mathrm{SAT}}=133 \mathrm{MHz}$ and with an effective isotropic radiated power (EIRP) of $-4 \mathrm{dBm} / \mathrm{MHz}$. The noise figures for the terrestrial and satellite transceivers are $9 \mathrm{~dB}$ and $1.2 \mathrm{~dB}$, respectively [16]. Users are also deployed in a random fashion (uniformly) throughout the terrestrial coverage area and they are assumed to have dual connection capability (terrestrial/satellite) [3]. Users' pilot transmit power $P_{t}^{\mathrm{MS}}=100 \mathrm{~mW}$ to enable the terrestrial channel estimation and the weighing parameter is set to $\rho=1$. Power allocation coefficients for both terrestrial and satellite users are determined using max-min optimization albeit on a per-segment fashion. Figure 2 depicts the average user rate (in $\mathrm{Mb} / \mathrm{s}$ ) for different users and under different infrastructure conditions when considering a pure CF-M-MIMO deployment and a LEO-enhanced CF-M-MIMO (hybrid) setup using Algorithm I to govern the terrestrial/satellite user split and assuming the use of $M=100$ APs in the CF-MMIMO segment. The solid lines depict the achievable user rates for each of these systems (CF-M-MIMO in black, and hybrid in blue) whereas, for completeness, the dashed lines represent the separate achievable rates
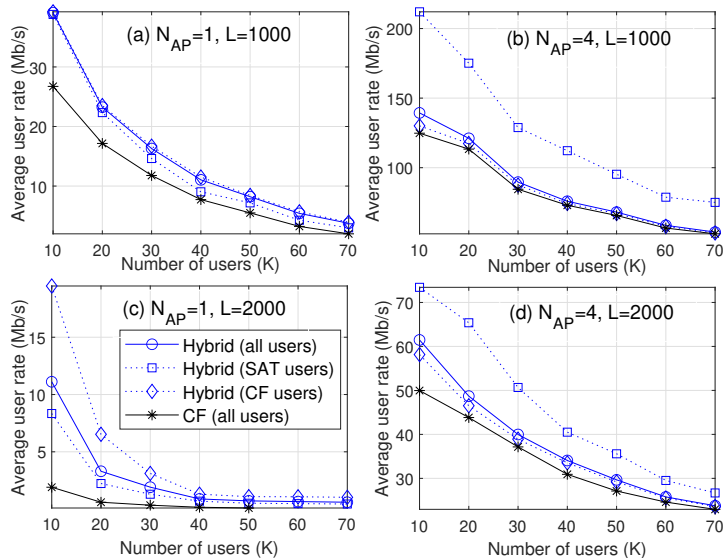

Fig. 2: Average user rate for different network loads under various infrastructure conditions for $M=100$.

of the two segments of the hybrid deployment. Two parameters are varied; the number of antennas at the APs $\left(N_{A P}\right)$ and the coverage square side $(L)$. The first and most general point to note is that the hybrid network always outperforms the CF-M-MIMO system, thus indicating that the user diverting algorithm works as intended. In particular, it is interesting to stress how the CF users in the hybrid system always outperform the pure CF-M-MIMO counterparts, a fact that reveals that diverting to the satellite segment those users that are limiting the max-min terrestrial performance is often an effective strategy. Starting now on the top left plot ((a), $\left.N_{A P}=1, L=1000\right)$, it can be seen how the hybrid network is able to offer very substantial throughput gains for any user load, a fact that contrasts with the situation depicted on the top right plot ((b), $\left.N_{A P}=4, L=1000\right)$, where the average overall gain becomes almost marginal. Interestingly, note how in the latter case, the satellitediverted users enjoy a phenomenal data rate, a trait indicating that the large bandwidth of the satellite segment is basically used by a single (two at most) diverted users. The message conveyed by these two figures is unequivocal: when a rich terrestrial infrastructure is available (i.e., dense deployment, complex APs), there is no benefit from granting users the possibility of a satellite connection. This conclusion is further reinforced by the bottom figures that re-examine the results but now considering a coverage four times larger than that on the top plots. In this case it can be seen how equipping the APs with just one antenna ((c), $\left.N_{A P}=1, L=2000\right)$ leads to an extremely poor performance of the CF-MMIMO even for lightly loaded situations. In contrast, by allowing the worst terrestrial users to be transferred to the satellite segment, the performance of the remaining 

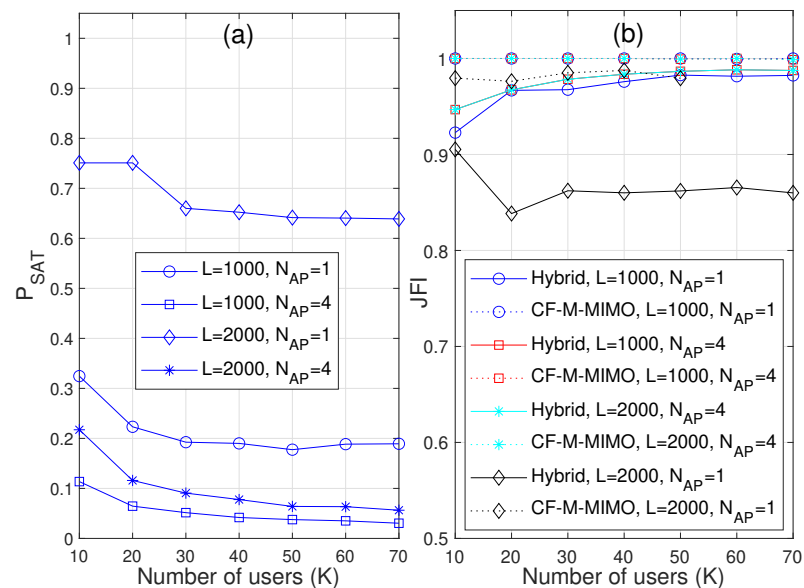

Fig. 3: (a) Probability of user diversion to the satellite segment $\left(P_{\mathrm{SAT}}\right)($ b) Jain's fairness index, for $M=100$.

terrestrial users is dramatically improved. Note that, in sheer contrast with (b), now a significant number of users are diverted and therefore have to share the satellite bandwidth, thus causing the satellite users to perform below average, a trend that is again reverted when more antennas are added to the APs ((d), $N_{A P}=4, L=$ 2000).

Figure 3(a) represents the probability for a terrestrial user to be diverted to the satellite segment $\left(P_{\mathrm{SAT}}\right)$. Note how under the poorest terrestrial infrastructure setup $\left(N_{A P}=1, L=2000\right)$, the probability of user diversion varies between 0.65 and 0.75 in an attempt to avoid the very bad operational conditions a pure CF-M-MIMO system offers. In contrast, as terrestrial infrastructure improves (more APs $/ \mathrm{m}^{2}$ and/or more antennas), diversion probability falls sharply. Figure 3(b) depicts the per-user rate Jain's fairness index (JFI) for both schemes (CF-M-MIMO and Hybrid) and the four considered scenarios in Fig. 2. The JFI, when applied on the throughput of $K$ users, is usually defined as $\mathbf{J}=$ $\left(\sum_{\forall k} R_{k}\right)^{2} /\left(K \sum_{k \in \mathcal{K}} R_{k}^{2}\right)$, and its value is constrained to the range $[1 / K, 1]$, with unity indicating perfect fairness. Noting that JFI values above 0.9 are indicative of very fair operation, results in Fig. 3(b) show how CFM-MIMO networks attain perfect user fairness $(\mathrm{J}=1)$ whereas the hybrid counterparts only pay a small penalty as a result of the separate optimization of the terrestrial and satellite segments. This fairness resilience is largely explained by the low diversion probability (hinted in Fig. 3(a)) that ensures that the performance of most users is still jointly optimized. The fairness drop for the hybrid configuration $\left(L=2000, N_{\mathrm{AP}}=1\right)$ has to be sought on the large probability of user diversion, a condition that effectively partitions users into two similar-sized groups each with rather different performance.

\section{CONCLUSION}

This paper has presented an integrated spaceterrestrial framework combining the benefits offered by an ultradense terrestrial deployment (CF-M-MIMO) with the large coverage of a LEO satellite segment. It has been shown that the max-min performance of the users being served can be substantially boosted specially in those scenarios where the ground segment is somewhat limited (insufficient AP density, single-antenna APs). Future work will investigate the role the satellite segment can play in coordinating several adjacent CF-M-MIMO segments and in servicing users transiting among them.

\section{REFERENCES}

[1] M. Jia and et al., "Broadband hybrid satellite-terrestrial communication systems based on cognitive radio toward 5G," IEEE Wireless Communications, vol. 23, no. 6, pp. 96-106, 2016.

[2] G. Giambene, S. Kota, and P. Pillai, "Satellite-5G integration: A network perspective," IEEE Network, vol. 32, no. 5, 2018.

[3] B. Di and et al., "Ultra-dense LEO: Integration of satellite access networks into 5G and beyond," IEEE Wireless Communications, vol. 26, no. 2, pp. 62-69, 2019.

[4] Y. Su and et al., "Broadband LEO satellite communications: Architectures and key technologies," IEEE Wireless Commun., vol. 26, no. 2, pp. 55-61, 2019.

[5] B. Di and et al., "Ultra-dense LEO: Integrating terrestrial-satellite networks into 5G and beyond for data offloading," IEEE Trans. on Wireless Commun., vol. 18, no. 1, 2018.

[6] J. Li and et al., "Energy efficiency and traffic offloading optimization in integrated satellite/terrestrial radio access networks," IEEE Trans. on Wireless Commun., vol. 19, no. 4, 2020.

[7] H. Q. Ngo and et al., "Cell-free massive MIMO versus small cells," IEEE Trans. on Wirel. Commun., vol. 16, no. 3, 2017.

[8] E. Nayebi and et al., "Precoding and power optimization in cellfree massive MIMO systems," IEEE Transactions on Wireless Communications, vol. 16, no. 7, pp. 4445-4459, 2017.

[9] H. Yang and T. L. Marzetta, "Massive MIMO with max-min power control in line-of-sight propagation environment," IEEE Trans. on Communications, vol. 65, no. 11, pp. 4685-4693, 2017.

[10] F. Riera-Palou and G. Femenias, "Decentralization issues in cellfree massive MIMO networks with zero-forcing precoding," in 57th IEEE Allerton Conf., 2019, pp. 521-527.

[11] R. Van der Breggen, "Why leo are key for telecom operators in search of growth," Satellite-Evolution, March, 2017.

[12] X. Zhu and et al., "Cooperative transmission in integrated terrestrial-satellite networks," IEEE Network, vol. 33, no. 3, pp. 204-210, 2019.

[13] G. Interdonato, P. Frenger, and E. G. Larsson, "Scalability aspects of cell-free massive MIMO," in IEEE ICC, 2019.

[14] A. Alvarez-Polegre, F. Riera-Palou, G. Femenias, and A. G. Armada, "Channel hardening in cell-free and user-centric massive MIMO networks with spatially correlated ricean fading," IEEE Access, vol. 8, pp. 139827-139845, 2020.

[15] E. Björnson, J. Hoydis, and L. Sanguinetti, "Massive MIMO networks: Spectral, energy, and hardware efficiency," Foundations and Trends ${ }^{\circledR}$ in Signal Processing, vol. 11, no. 3-4, 2017.

[16] 3GPP, "Study on new radio (NR) to support non-terrestrial networks(Release 15)," 3GPP TR 38.811 (v15.3.0), July 2020.

[17] F. Riera-Palou and G. Femenias, "Trade-offs in cell-free massive MIMO networks: Precoding, power allocation and scheduling," in 14th IEEE TELSIKS conference, 2019, pp. 158-165. 